\title{
Orbital magnetic field effects in spin liquid with spinon Fermi sea: Possible application to $\kappa-(\mathrm{ET})_{2} \mathrm{Cu}_{2}(\mathrm{CN})_{3}$
}

\author{
Olexei I. Motrunich \\ Kavli Institute for Theoretical Physics, University of California, Santa Barbara, California 93106-4030, USA \\ (Received 28 November 2005; revised manuscript received 13 February 2006; published 19 April 2006)
}

\begin{abstract}
We consider orbital magnetic field effects in a spin liquid phase of a half-filled triangular lattice Hubbard system close to the Mott transition, continuing an earlier exploration of a state with spinon Fermi surface. Starting from the Hubbard model and focusing on the insulator side, we derive an effective spin Hamiltonian up to four-spin exchanges in the presence of magnetic field, and find that the magnetic field couples linearly to spin chirality on the triangles. The latter corresponds to a flux of an internal gauge field in a gauge theory description of the spin liquid, and therefore a static internal flux is induced. A quantitative estimate of the effect is obtained using a spinon mean-field analysis, where we find that this orbital field experienced by the spinons is comparable to or even larger than the applied field. We further argue that because the stiffness of the emergent internal gauge field is very small, such a spinon-gauge system is strongly susceptible at low temperatures to an instability of the homogeneous state due to strong Landau level quantization for spinons. This instability is reminiscent of the so-called strong magnetic interaction regime in metals with the usual electromagnetic field, but we estimate that the corresponding temperature-magnetic-field range is significantly broader in the spinon-gauge system.
\end{abstract}

DOI: 10.1103/PhysRevB.73.155115 PACS number(s): 75.10.Jm, 75.50.Ee, 75.40. - s, 74.70.Kn

\section{INTRODUCTION}

Experimental studies ${ }^{1-3}$ of the quasi-two-dimensional organic material $\kappa-(\mathrm{ET})_{2} \mathrm{Cu}_{2}(\mathrm{CN})_{3}$ strongly suggest a spin liquid state in the insulating phase at ambient pressure. $\kappa-(\mathrm{ET})_{2} \mathrm{Cu}_{2}(\mathrm{CN})_{3}$ is a strongly correlated half-filled Hubbard system on an almost isotropic triangular lattice. The material is just near the boundary of the insulator-metal transition. ${ }^{1-7}$ It is an insulator at ambient pressure with a charge gap of order $350 \mathrm{~K}$, and shows no signs of magnetic ordering down to $32 \mathrm{mK}$ despite a relatively large exchange coupling $J$ $\sim 250 \mathrm{~K}$. Experiments find that this spin liquid maintains a finite susceptibility and a finite $1 /\left(T_{1} T\right)$ for the ${ }^{13} \mathrm{C}$ nuclear spin relaxation rate down to low temperatures, ${ }^{1,5}$ and also that this insulator has large spin entropy.,3

These observations and related numerical studies ${ }^{7,8}$ led the present author ${ }^{9}$ and also the authors of Ref. 10 to propose a spin liquid state with spinon Fermi surface as a likely candidate. In Ref. 9, this state was shown to be energetically favored in a spin model with Heisenberg and ring exchanges appropriate for the description of the insulator, while Ref. 10 used the mean-field slave-particle approach and also derived an effective gauge theory description of the proposed spin liquid.

Here, we examine the response of this spin liquid to strong magnetic fields. One motivation is to look for possible direct probes of the spinon Fermi surface similar to the ones used in metals such as magneto-oscillations or magnetoacoustic resonance. Another motivation is the observations in Refs. 3 and 5 of significant broadening of the ${ }^{13} \mathrm{C}$ NMR line in a large magnetic field up to $9 \mathrm{~T}$ at low temperatures below 10-30 K. Systematic studies ${ }^{3}$ reveal that the lines broaden symmetrically and the broadening increases with increasing field and also with decreasing temperature. At the present, it is not clear whether this anomalous behavior is intrinsic, or is the result of extrinsic disorder effects in the critical spin liquid.

A simple intuition about the Mott insulator would be that the charge motion is suppressed and only spin degrees of freedom couple to the magnetic field. If this were the case, then one would not expect strong intrinsic effects in the presence of the magnetic field. In particular, in the spin liquid state with spinon Fermi surface and just the Zeeman spin coupling to the applied field one would expect only Pauli spin paramagnetism. We argue, however, that orbital effects need to be carefully included when analyzing the response of the spin liquid in $\kappa-(\mathrm{ET})_{2} \mathrm{Cu}_{2}(\mathrm{CN})_{3}$. This is because charge fluctuations, which become more prominent in the vicinity of the insulator-metal transition, also induce an effective "orbital" coupling to the magnetic field. The effect of such coupling on the proposed spinon Fermi sea state is further amplified by the fact that this phase itself is stabilized against other competing spin liquids or the antiferromagnetically ordered state by charge fluctuations that produce the four-spin ring exchange terms. ${ }^{9,10}$

First, we show that in the presence of the external magnetic field the spinons effectively experience an "internal" orbital field that is comparable to and maybe even larger than the applied field. This is despite the fact that spinons do not transport electrical charge, but follows when we derive an effective spin Hamiltonian from electronic degrees of freedom in the presence of the applied magnetic field. We find that the magnetic field couples linearly to the spin chirality on the elementary triangles. The effective description of the spin liquid state contains spinons coupled to a dynamically generated "internal" gauge field. The physical meaning of the flux of the internal gauge field is precisely the spin chirality, and the external magnetic field therefore induces a static internal flux seen by the spinons that is comparable to the applied field. 
Second, we argue that because the stiffness of the internal gauge field is very small (see Appendix $\mathrm{C}$ for numerical estimates), the response of the spinon-gauge field system changes dramatically at low temperatures such that the Landau quantization of the spinons in the static internal field is not smeared by the temperature. These effects are similar to strong electronic magnetism in quantizing field at low temperatures familiar in magneto-oscillation studies of metals $^{11-13}$ (which we also review in Appendix D). In particular, the homogeneous state with continuously varying internal field becomes unstable below some temperature, which for the $\kappa$ - $(\mathrm{ET})_{2} \mathrm{Cu}_{2}(\mathrm{CN})_{3}$ spin liquid we estimate to be several kelvin in typical laboratory fields. The instability regime is significantly wider in the spinon-gauge system than in metals because the internal gauge field stiffness is so much smaller than that of the physical electromagnetic field. Crudely, the spinon states with integer Landau level filling are more stable than the states with a continuously varying filling, and it becomes advantageous for the internal gauge field to adjust itself discontinuously to achieve this. This instability also preempts the possibility of a direct observation of the spinon Fermi surface using magneto-oscillation probes, but can be viewed as an extreme manifestation of such magneto-oscillations.

The objective of the present paper is to characterize the above two predictions in the spinon-gauge system focusing on the $\kappa-(\mathrm{ET})_{2} \mathrm{Cu}_{2}(\mathrm{CN})_{3}$ material. The paper is organized as follows. In Sec. II (and also Appendix A), we describe the effective spin Hamiltonian derived from the triangular lattice Hubbard model in the magnetic field. In the main part of the paper, Sec. III, we perform a spinon mean-field study of the effective Hamiltonian. We start with a review of the system in zero field ${ }^{9}$ and progressively add the needed ingredients for the discussion of the response to the magnetic field such as the appearance of the static internal gauge field and the importance of the spinon Landau level quantization at low temperatures. We then argue that a homogeneous spin liquid state with continuously varying static internal flux becomes unstable at low but experimentally relevant temperatures of few kelvin. We finalize this section with a more careful discussion of the physical setting in the real system. Helpful connections with the gauge theory description and analogies with magnetic interaction effects in metals are summarized in Appendixes $\mathrm{C}$ and $\mathrm{D}$. Throughout, we use the $\kappa$ - $(\mathrm{ET})_{2} \mathrm{Cu}_{2}(\mathrm{CN})_{3}$ parameters as a guide for the relevant questions. In Sec. IV, we collect the main estimates for the $\kappa$-(ET) $)_{2} \mathrm{Cu}_{2}(\mathrm{CN})_{3}$ material and consider some experimental aspects.

\section{RING EXCHANGE HAMILTONIAN IN THE PRESENCE OF MAGNETIC FIELD}

The approach adopted in Ref. 9 and pursued here is to focus on the spin degrees of freedom when describing the insulating state. This is achieved by considering an effective spin Hamiltonian obtained from the microscopic Hubbard model by a canonical transformation that projects out the double occupancy. The importance of the charge fluctuations is retained in the form of more complicated multispin ex- changes. The spin system is still more amenable to analysis because there is much less disproportion between the relevant energy scales and the couplings in the effective Hamiltonian, unlike in the original Hubbard model.

The effective Hamiltonian to order $t^{4} / U^{3}$ on the isotropic triangular lattice reads (see also Appendix A)

$$
\begin{aligned}
& \hat{H}_{\text {eff }}=J_{2} \sum_{\bullet \bullet} P_{12}+J_{4} \sum_{\bullet}\left(P_{1234}+\text { H.c. }\right) \\
& +J^{\prime \prime} \sum_{\langle\langle i j\rangle\rangle} \mathbf{S}_{i} \cdot \mathbf{S}_{j}+J^{\prime \prime \prime} \sum_{\langle\langle i j\rangle\rangle)} \mathbf{S}_{i} \cdot \mathbf{S}_{j} \\
& +\Phi_{\triangle}^{\mathrm{ext}} J_{3} \sum_{\Omega} i\left(P_{123}-\text { H.c. }\right) \text {. }
\end{aligned}
$$

Here we use multispin exchange operators defined as $P_{1,2, \ldots, n}:\left|\sigma_{1}, \sigma_{2}, \ldots, \sigma_{n}\right\rangle \rightarrow\left|\sigma_{n}, \sigma_{1}, \ldots, \sigma_{n-1}\right\rangle$. For two spins, this reduces to the familiar Heisenberg exchange $P_{12}=P_{12}^{\dagger}$ $=2 S_{1} \cdot S_{2}+\frac{1}{2}$. The first two lines give the effective Hamiltonian in the absence of the magnetic field. As discussed in Ref. 9, important terms in this Hamiltonian are the nearestneighbor two-spin exchanges and the ring exchanges around the rhombi of the triangular lattice. The corresponding coupling constants are given in terms of the Hubbard model parameters as

$$
J_{2}=\frac{2 t^{2}}{U}\left(1-\frac{32 t^{2}}{U^{2}}\right), \quad J_{4}=\frac{20 t^{4}}{U^{3}} .
$$

The second- and third-neighbor Heisenberg exchanges $J^{\prime \prime}=$ $-16 t^{4} / U^{3}$ and $J^{\prime \prime \prime}=4 t^{4} / U^{3}$, though nominally of the same order as the ring exchange coupling, were argued to play a minor role because of the weak correlations between such further-neighbor spins in the magnetically disordered state.

The last line in Eq. (1) shows a new term that appears at order $t^{3} / U^{2}$ in the presence of the magnetic field. This term involves three-spin exchanges around the elementary triangles and is proportional to the enclosed flux with the coupling constant

$$
J_{3}=\frac{6 t^{3}}{U^{2}} .
$$

The dimensionless flux is $\Phi_{\Delta}^{\mathrm{ext}}=e B A_{\Delta} / \hbar c$, where $B$ is the field and $A_{\Delta}$ is the area. The Hamiltonian Eq. (1) is written to linear order in the external flux, which is assumed to be small, $\Phi_{\Delta}^{\text {ext }} \ll 1$. Each triangle is counted once and is traversed in the same direction. We can write the $J_{3}$ term more explicitly as $\sin \left(\Phi_{123}^{\text {ext }}\right) i\left(P_{123}-\right.$ H.c. $)$, where $\Phi_{123}^{\text {ext }}$ is the complex phase of the loop product $t_{12} t_{23} t_{31}$ of the electron hopping amplitudes in the field. In general, each contribution from an exchange path that encloses flux is affected by the magnetic field (cf. Appendix A). However, the couplings $J_{2}$, $J_{4}, J^{\prime \prime}$, and $J^{\prime \prime \prime}$ are modified only at quadratic order in $\Phi^{\text {ext }}$. The exhibited three-spin terms represent the full effect linear in $\Phi^{\text {ext }}$ to order $t^{4} / U^{3}$. The effective Hamiltonian contains only terms $P_{12},\left(P_{1234}+\right.$ H.c. $)$, and $i\left(P_{123}-\right.$ H.c. $)$, which was achieved with the help of the following identities valid in the spin- $1 / 2$ case: 


$$
\begin{gathered}
P_{123}+\text { H.c. }=P_{12}+P_{23}+P_{31}-1, \\
P_{1234}-\text { H.c. }=\frac{1}{2}\left(P_{123}+P_{234}+P_{341}+P_{412}-\text { H.c. }\right) .
\end{gathered}
$$

The three-spin operator $i\left(P_{123}-\right.$ H.c. $)$ has a simple physical meaning - it represents the spin chirality

$$
i\left(P_{123}-\text { H.c. }\right)=-4 S_{1} \cdot S_{2} \times S_{3} .
$$

Thus, the external field couples linearly to the spin chirality and therefore induces such chirality density in the system.

In the above discussion, we have not mentioned the original Zeeman coupling of the electrons to the magnetic field. Since the Zeeman term involves the conserved total $S_{z}$, we can simply reinstate it in the final effective spin Hamiltonian.

For $\kappa$ - $(\mathrm{ET})_{2} \mathrm{Cu}_{2}(\mathrm{CN})_{3}$, we use $t=55 \mathrm{meV}$ and $U / t=8.2$ to obtain $J_{2} \simeq 7 \mathrm{meV}, J_{4} / J_{2} \simeq 0.3$, and $J_{3} / J_{2} \simeq 0.7$; the Heisenberg exchange coupling is $J=2 J_{2} \simeq 160 \mathrm{~K}$, which is comparable with the estimate $J \simeq 250 \mathrm{~K}$ in Ref. 1 .

To summarize, when charge fluctuations are significant, the effective spin Hamiltonian in the presence of the applied magnetic field is modified beyond the direct Zeeman term and contains a linear coupling of the spin chirality to the external field. This orbital effect is important when considering the response of such insulator to the magnetic field.

\section{SPINON MEAN-FIELD DESCRIPTION}

\section{A. General setting}

In Ref. 9 we considered the situation with no magnetic field and argued that the four-spin ring exchanges stabilize the spin liquid state with spinon Fermi surface. This state can be viewed as a Gutzwiller projection of a fermionic meanfield state in which spinons hop on the triangular lattice. We performed a direct variational wave function study, and also provided an intuitive mean-field argument for the stabilization of this state.

A similar mean-field treatment is pursued here to understand the effects of the magnetic field. To simplify the discussion, we will focus on the $J_{2}-J_{3}-J_{4}$ terms in $H_{\text {eff }}$. As mentioned earlier, the second- and third-neighbor Heisenberg exchanges are not expected to have significant effect.

Each spin-1/2 is represented in terms of two "spinon" operators $f_{\uparrow}, f_{\downarrow}$, with the occupancy constraint ${ }^{14,15}$

$$
f_{r \sigma}^{\dagger} f_{r \sigma}=1 \text {. }
$$

In this representation, the $J_{2}-J_{3}-J_{4}$ Hamiltonian reads

$$
\begin{aligned}
\hat{H}= & J_{2} \sum_{\text {links }}\left(f_{1 \alpha}^{\dagger} f_{1 \beta}\right)\left(f_{2 \beta}^{\dagger} f_{2 \alpha}\right) \\
& +J_{4} \sum_{\text {rhombi }}\left[\left(f_{1 \alpha}^{\dagger} f_{1 \beta}\right)\left(f_{2 \beta}^{\dagger} f_{2 \gamma}\right)\left(f_{3 \gamma}^{\dagger} f_{3 \delta}\right)\left(f_{4 \delta}^{\dagger} f_{4 \alpha}\right)+\text { H.c. }\right] \\
& +J_{3} \sum_{\text {triangles }} \sin \left(\Phi_{123}^{\mathrm{ext}}\right) i\left[\left(f_{1 \alpha}^{\dagger} f_{1 \beta}\right)\left(f_{2 \beta}^{\dagger} f_{2 \gamma}\right)\left(f_{3 \gamma}^{\dagger} f_{3 \alpha}\right)-\text { H.c. }\right] .
\end{aligned}
$$

For clarity, we use the form of the $J_{3}$ term valid for general $\Phi^{\text {ext }}$ (cf. Appendix A), even though we are interested in the case of small $\Phi_{\Delta}^{\text {ext }} \ll 1$.
We consider trial spinon hopping Hamiltonians ${ }^{15,16}$

$$
\hat{H}_{\text {trial }}=-\sum_{\left\langle r r^{\prime}\right\rangle}\left(t_{r r^{\prime}}^{\text {spinon }} f_{r \alpha}^{\dagger} f_{r^{\prime} \alpha}+\text { H.c. }\right)-\sum_{r} \mu_{r} f_{r \alpha}^{\dagger} f_{r \alpha},
$$

with general hopping amplitudes $t_{r r^{\prime}}^{\text {spinon }}=\left(t_{r^{\prime} r}^{\text {spinon }}\right)^{*}$. The occupancy constraints are implemented on average by the appropriate chemical potentials $\mu_{r}$. The mean-field scheme that we use here is to evaluate the above exchange terms by contracting only the fermions with the same spin index. This approach can be justified in a large- $N$ fermionic generalization, since any other contraction is down by a factor of $1 / N$. While the present $N=2$ is not large, the other contractions do not introduce qualitatively new terms, and this scheme is expected to capture the relevant physics rather well. The mean-field energy is

$$
\begin{aligned}
E_{\mathrm{mf}}= & -4 g_{2} J_{2} \sum_{\text {links }}\left|\chi_{12}\right|^{2}-16 g_{4} J_{4} \sum_{\text {rhombi }}\left(\chi_{12} \chi_{23} \chi_{34} \chi_{41}+\text { c.c. }\right) \\
& -8 g_{3} J_{3} \sum_{\text {triangles }} \sin \left(\Phi_{123}^{\mathrm{ext}}\right)(-i)\left(\chi_{12} \chi_{23} \chi_{31}-\text { c.c. }\right)
\end{aligned}
$$

Here $\chi_{r r^{\prime}} \equiv\left\langle f_{r^{\prime}}^{\dagger} f_{r}\right\rangle$ are link expectation values evaluated for one spin species, and the powers-of-2 numerical factors appear from the spin summations. We also introduce phenomenological renormalization factors $g_{2}, g_{4}$, and $g_{3}$, to keep us aware of the schematic character of the mean-field treatment. In a more quantitative treatment, these factors can be estimated by matching to numerical evaluations with the Gutzwiller-projected wave functions. This is done in Appendix B, where we find that $g_{2} \simeq 1.7, g_{4} \simeq 8$, and $g_{3} \simeq 5$ reproduce fairly well such direct trial wave function computations.

The mean-field energy is to be minimized over the trial spinon hopping amplitudes $t_{r r^{\prime}}^{\text {spinon }}$. We focus on the states that describe translationally invariant spin liquids. ${ }^{15}$ These are the projected Fermi sea state with real hopping amplitudes and the so-called flux states with complex $t_{r r^{\prime}}^{\text {spinon }}$ realizing nontrivial "internal" fluxes through the hopping loops. The flux can be either uniform or have a staggered pattern. The projected Fermi sea state is a special case with zero flux. Here, we are primarily interested in the uniform flux states since these are natural candidates in the presence of the external magnetic field if one starts with the zero-flux state in the absence of the magnetic field.

The mean-field energy per site for a uniform flux state is

$$
\begin{aligned}
\epsilon_{\mathrm{mf}}= & -12 g_{2} J_{2}\left|\chi_{\phi}\right|^{2}-96 g_{4} J_{4}\left|\chi_{\phi}\right|^{4} \cos \left(\phi_{1234}^{\mathrm{int}}\right) \\
& -32 g_{3} J_{3}\left|\chi_{\phi}\right|^{3} \sin \left(\Phi_{123}^{\mathrm{ext}}\right) \sin \left(\phi_{123}^{\mathrm{int}}\right) .
\end{aligned}
$$

Here, $\chi_{r r^{\prime}}=\left|\chi_{\phi}\right| \exp \left(i a_{r r^{\prime}}\right)$ and $\phi^{\text {int }}$ is the flux of the "internal" gauge field $a_{r r^{\prime}}$. Note that the link expectation values $\chi_{r r^{\prime}}$ obtain the same flux pattern as the input amplitudes $t_{r r^{\prime}}^{\text {spinon }}$. The gauge theory language is explained in Appendix C.

\section{B. Review of the zero-field case}

Let us first consider $\Phi^{\text {ext }}=0$ following Ref. 9. We find that for small $g_{4} J_{4} /\left(g_{2} J_{2}\right) \leqslant 0.69$, the lowest-energy state has $\phi_{\triangle}=\pi / 2$ flux through each triangle, while for larger ring 


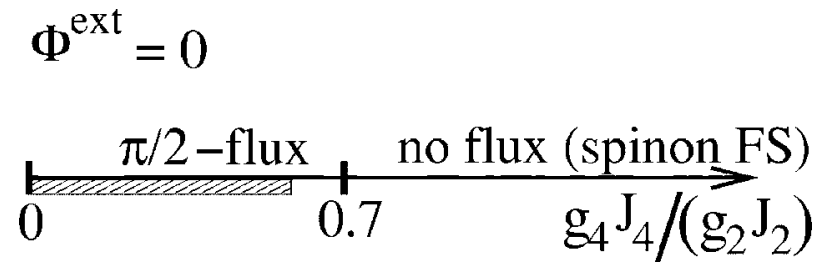

FIG. 1. "Phase diagram" from the mean-field energy optimization over translationally invariant states in the absence of the magnetic field. In the hatched region, the zero-flux state is also unstable against introducing small internal flux. For the $\kappa-(\mathrm{ET})_{2} \mathrm{Cu}_{2}(\mathrm{CN})_{3}$ parameters, we estimate $g_{4} J_{4} /\left(g_{2} J_{2}\right) \simeq 1.2-1.5$.

exchanges the best state has zero flux (see Fig. 1). The corresponding numerical values of $\left|\chi_{\phi}\right|$ for the half-filled triangular lattice are

$$
\left|\chi_{\phi=\pi / 2}\right|=0.2002, \quad\left|\chi_{\phi=0}\right|=0.1647 .
$$

It is known ${ }^{17}$ that flux states have large absolute value $\left|\chi_{\phi}\right|$ and therefore good Heisenberg energies. On the other hand, the ring exchanges are directly sensitive to the placket fluxes and dislike any fluxes, as can be seen from Eq. (10). This is why the zero-flux state is stabilized for large $J_{4} / J_{2}$. As explained in Ref. 9, we do not consider so-called dimer states even though formally for fixed $g_{2} J_{2}$ these have the lowest mean-field energy in the range $g_{4} J_{4} /\left(g_{2} J_{2}\right) \leqslant 2.4$. Our reasoning is that $J_{2}$ is fixed, and to go from the mean-field evaluations to the projected wave functions, the renormalization factor $g_{2}$ is larger for the translationally invariant states.

For later convenience, Fig. 2 shows the behavior of $\left|\chi_{\phi}\right|$ evaluated numerically for the triangular lattice flux states. It is useful to note the enveloping function for small fluxes, which we find to be

$$
\left|\chi_{\phi}\right| \leqslant\left|\chi_{0}\right|\left(1+c \phi_{\Delta}^{2}\right)
$$

with $c \approx 0.1$. In particular, it follows that for small $g_{4} J_{4} /\left(g_{2} J_{2}\right) \leqslant 0.56$ - hatched region in Fig. 1-the zero-flux state is also unstable against introducing weak fluxes (while the $\pi / 2$-flux state is the global translationally-invariant minimum until a somewhat larger value of 0.69).

A direct Gutzwiller wave function study gives that the zero-flux state has lower energy than the $\pi / 2$-flux state for $J_{4} \geqslant 0.145 J_{2}$. From this and similar comparisons we estimate that $g_{4} / g_{2}$ is roughly 4 to 5 - see Appendix B for more details.

In the $\kappa-(\mathrm{ET})_{2} \mathrm{Cu}_{2}(\mathrm{CN})_{3}$ compound, we have $J_{4} / J_{2} \simeq 0.3$, which is not far from the regime where it would be advantageous to spontaneously generate such internal flux. We therefore reason that the spinon Fermi sea state in the $\kappa-(\mathrm{ET})_{2} \mathrm{Cu}_{2}(\mathrm{CN})_{3}$ compound is rather susceptible to the internal flux generation. In particular, we expect some enhancement in the response to the external magnetic field, to which we now turn.

\section{Mean field over homogeneous flux states in the presence of the magnetic field}

Consider the mean-field energy Eq. (10) with nonzero but small $\Phi^{\text {ext }}$. Let us first try the enveloping function Eq. (12)

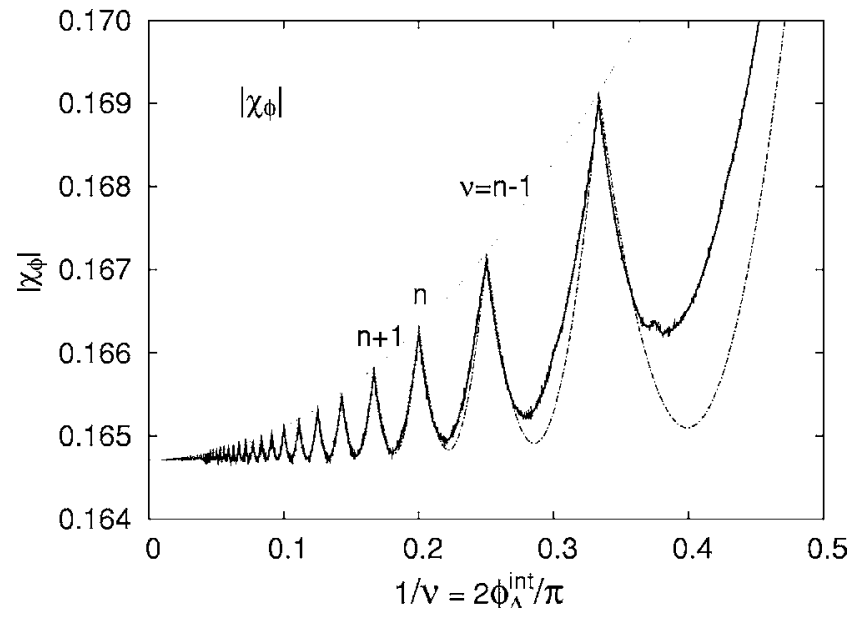

FIG. 2. Mean-field numerical data for uniform flux states on the half-filled triangular lattice. The figure shows the absolute value of the bond expectation value $\left|\chi_{\phi}\right| \equiv\left|\left\langle f_{r}^{\dagger} f_{r}\right\rangle\right|$ as a function of the spinon filling factor $\nu$ with respect to the internal flux (one spin species is considered). The dotted line is the enveloping function Eq. (14), while the dash-dotted line is the full model function Eq. (15). The data plotted correspond to rather large $\phi^{\text {int }}$, but the same behavior is expected to continue for small fluxes.

for small $\phi^{\text {int }}$. Expanding $\epsilon_{\mathrm{mf}}$ to quadratic order in $\phi^{\text {int }}$ and minimizing over the internal flux, we find

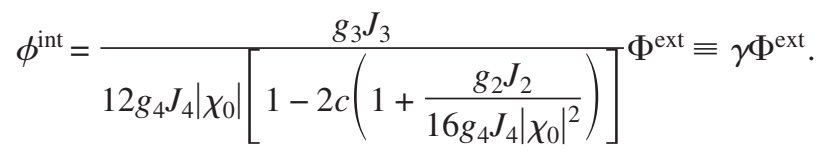

The different ingredients have the following physical origin. The $J_{3}$ in the numerator represents the coupling of the magnetic field to the internal gauge flux through the corresponding three-spin term. On the other hand, the $J_{4}$ in the denominator represents the stiffness of the internal gauge field that originates from the four-spin exchanges. For the $\kappa-(\mathrm{ET})_{2} \mathrm{Cu}_{2}(\mathrm{CN})_{3}$ parameters, we estimate $g_{3} J_{3} /\left(12 g_{4} J_{4}\left|\chi_{0}\right|\right) \simeq 0.7$. The term in the square brackets in the denominator reflects the enhanced susceptibility to the fluxes discussed above; it can be also viewed as a suppression of the internal gauge field stiffness. In particular, when this term goes to zero, the spinon Fermi sea state becomes unstable to spontaneous flux generation even in the absence of the external field. Given the proximity of the competing states, it is therefore reasonable to estimate this number to be of order one-half, so for the $\kappa-(\mathrm{ET})_{2} \mathrm{Cu}_{2}(\mathrm{CN})_{3}$ material we roughly estimate

$$
\gamma_{\kappa-(\mathrm{ET})_{2} \mathrm{Cu}_{2}(\mathrm{CN})_{3}} \simeq 1-2 .
$$

Thus, we conclude that due to the triangular ring exchanges and the proximity to the flux (or antiferromagnetic) instability, the effective orbital field seen by the spinons is comparable and can even be larger than the applied magnetic field.

We now examine the mean-field energy Eq. (10) more carefully. In the presence of the static internal gauge flux, the spinon spectrum consists of Landau bands. For either spin 


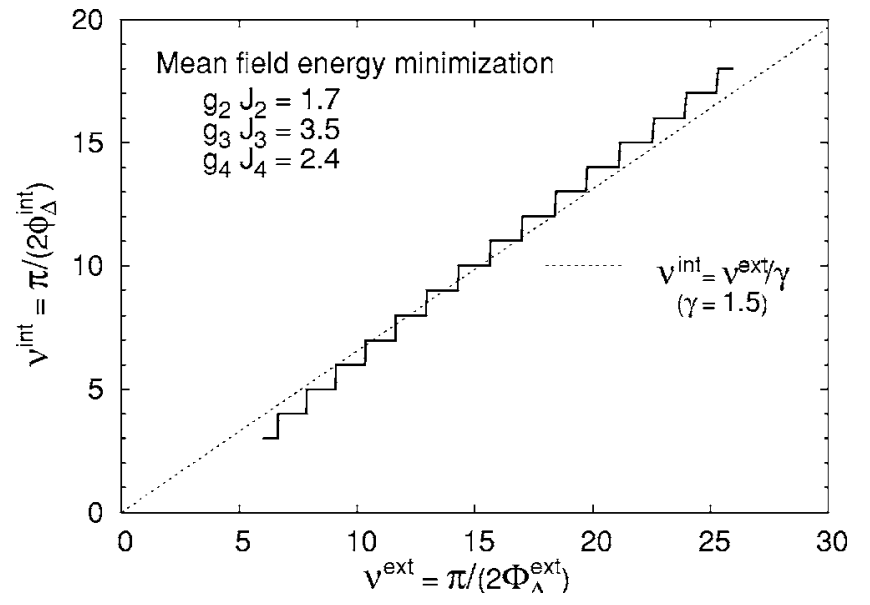

FIG. 3. Result of the mean-field energy minimization with respect to $\phi^{\text {int }}$ in the presence of the magnetic field at zero temperature. The optimal spinon filling factor $\nu^{\text {int }}$ is plotted vs the nominal filling factor $\nu^{\text {ext }}$ for the applied magnetic field. The figure shows a sequence of first-order transitions with $\nu^{\text {int }}$ stepping through integer values. The parameters roughly correspond to our estimates for the $\kappa-(\mathrm{ET})_{2} \mathrm{Cu}_{2}(\mathrm{CN})_{3}$ from Sec. II, with appropriate renormalization factors from Appendix B. The dotted line shows the overall trend Eq. (13). The data plotted correspond to unrealistically large fields, but the same behavior is expected to continue for smaller laboratory fields.

species, the filling factor of these Landau levels is $\nu$ $=\pi /\left(2 \phi_{\Delta}^{\text {int }}\right)$, and the flux states are special when $\nu$ is integer. Thus, the bond expectation value $\left|\chi_{\phi}\right|$ has upward-pointing cusps at these fluxes as can be seen in Fig. 2. The states with integer $\nu$ are therefore expected to be more stable. In fact, when the mean-field energy is minimized with respect to $\phi^{\text {int }}$ using the material parameters quoted above, we find that the optimal such flux does not change continuously but instead goes through this discrete set corresponding to the integer filling of the spinon Landau levels. This is shown in Fig. 3. The overall magnitude is still given by Eq. (13).

Let us exhibit the structure of the discussed mean field in a more transparent way. The reason behind the elementary manipulations below is to draw a connection with the socalled strong magnetic interaction effects in the magnetooscillation studies in metals. These are reviewed in Appendix $\mathrm{D}$, which contains useful interpretations of the expressions below. At the expense of being repetitious, what follows will allow us to estimate appropriate effective parameters, which we will then use in our discussion of the spinon system at finite temperature.

First of all, we model the behavior of $\left|\chi_{\phi}\right|$, Fig. 2, as

$$
\left|\chi_{\phi}\right|=\left|\chi_{0}\right|\left(1+c \phi_{\Delta}^{2}\right)-\Xi_{\phi}^{\text {osc }}
$$

where we separate the envelope Eq. (12), which is the band structure effect, and the oscillating part $\Xi_{\phi}^{\text {osc }}$ due to the discreteness of the spinon Landau levels. The latter is well approximated by the familiar expression for the oscillating piece of the energy of the continuum Landau problem,

$$
\Xi_{\phi}^{\text {osc }} \propto \nu^{-2}(\nu-k)(k+1-\nu)
$$

for the filling factor $\nu$ between integers $k$ and $k+1$. This approximation is also plotted in Fig. 2 with a suitably chosen numerical amplitude for $\Xi_{\phi}^{\text {osc }}$. In particular, from the numerical data we estimate the cyclotron mass for the half-filled triangular lattice band to be $m_{c} \approx t_{\text {spinon }}^{-1}$ in units with the lattice spacing set to 1 , while the corresponding cyclotron frequency at filling $\nu$ is

$$
\hbar \omega_{c} \approx 3.57 t_{\text {spinon }} / \nu .
$$

Returning to the mean-field energy Eq. (10), we expand to quadratic order in $\phi^{\text {int }}$ bearing in mind that the oscillating piece $\Xi_{\phi}^{\text {osc }}$ has an overall magnitude $\left(\phi^{\text {int }}\right)^{2}$ :

$$
\begin{gathered}
\epsilon_{\mathrm{mf}}=\kappa_{4}\left[\left(\phi_{\Delta}^{\mathrm{int}}\right)^{2}-2 \gamma \Phi_{\Delta}^{\mathrm{ext}} \phi_{\Delta}^{\mathrm{int}}\right]+\epsilon^{\mathrm{osc}}, \\
\epsilon^{\mathrm{osc}}=\kappa_{4} y^{\prime} \frac{\Xi_{\phi}^{\mathrm{osc}}}{\left|\chi_{0}\right|}=12 t_{\text {spinon }} \Xi_{\phi}^{\mathrm{osc}},
\end{gathered}
$$

with the effective parameters

$$
\begin{gathered}
\kappa_{4}=192 g_{4} J_{4}\left|\chi_{0}\right|^{4}(1-2 c y), \\
t_{\text {spinon }}=2 g_{2} J_{2}\left|\chi_{0}\right|+32 g_{4} J_{4}\left|\chi_{0}\right|^{3} .
\end{gathered}
$$

Here $\gamma$ is defined in Eq. (13); $y \equiv 1+g_{2} J_{2} /\left(16 g_{4} J_{4}\left|\chi_{0}\right|^{2}\right)$; and $y^{\prime} \equiv 2 y /(1-2 c y)$. We recall that the energy here is per triangular lattice site, and see that $\kappa_{4}$ is the corresponding measure of the stiffness of the internal gauge field-see Appendix C. We also see explicitly how the magnetic field acts to induce the internal gauge flux. On the other hand, using Eq. (15), we can interpret $\epsilon^{\text {osc }}$ as an oscillating piece of some fermion kinetic energy of spinful fermions hopping on the triangular lattice with amplitude $t_{\text {spinon }}$. It is useful to remember that we are analyzing the mean-field energy Eq. (10), and from this we are separating out what looks like an effective spinon kinetic energy. The division of the mean-field energy into the gauge field and the spinon parts is somewhat arbitrary, but represents a useful separation by the character of their dependence on $\phi^{\text {int }}$. In particular, we can now see the similarity with Eq. (D3) and thus connect with the studies of magnetic interaction effects in metals summarized in Appendix $\mathrm{D}$. The above equations constitute the main result of this section.

To emphasize the variational energetics character of the mean-field procedure and the intrinsically common origin of the gauge field and the spinon parts, we do not use such suggestive separation explicitly in the treatment below. A formal condition for an extremum of $\epsilon_{\mathrm{mf}}$ reads

$$
\gamma \Phi_{\Delta}^{\text {ext }}=\phi_{\Delta}^{\text {int }}+\frac{y^{\prime}}{2\left|\chi_{0}\right|} \frac{\partial \Xi_{\phi}^{\text {osc }}}{\partial \phi_{\Delta}^{\text {int }}} \equiv \phi_{\Delta}^{\text {int }}-\mathfrak{M}^{\text {osc }}\left[\phi_{\Delta}^{\text {int }}\right]
$$

which can be solved graphically by plotting the right-hand side as a function of $\phi^{\text {int }}$ and seeking crossings with the horizontal line $\gamma \Phi^{\text {ext }}$. This can be compared with Eq. (D4) in Appendix D. Here we remark that the oscillations of the "magnetization" $\mathfrak{M}^{\text {osc }}$ have roughly the sawtooth pattern familiar in two dimensions, with the amplitude which can be estimated from the data in Fig. 2. Note that the internal 
gauge field stiffness is implicitly included in $\mathfrak{M}^{\text {osc }}$, cf. Eq. (D4). We estimate that the corresponding $\left|\mathfrak{M}^{\text {osc }}\right|_{\max }$ is as large as 1. In particular, we conclude that at zero temperature essentially each oscillation period supplies such graphical solutions of Eq. (22). Solutions are found even in the region with $\nu \sim 1$ (corresponding to very large internal flux) even when $\Phi^{\text {ext }}$ is small. Remembering the cusps in $\left|\chi_{\phi}\right|$, this means that each integer filling gives a locally stable minimum, which we can also verify directly by plotting $\epsilon_{\mathrm{mf}}$ as a function of $\phi^{\text {int }}$. The global minimum is given by an integer filling near that corresponding to Eq. (13). This provides a more complete description of our direct minimization result Fig. 3 at zero temperature, and is useful when generalizing to finite temperature.

The multiple solutions signify an instability of the flux states with continuously varying $\phi^{\text {int }}$. The instability occurs whenever the derivative of the right-hand side of Eq. (24) becomes negative:

$$
\frac{\partial \mathfrak{M}^{\mathrm{osc}}}{\partial \phi_{\Delta}^{\text {int }}}>1 \Rightarrow \nu^{2}\left|\mathfrak{M}^{\mathrm{osc}}\right|_{\max } \gtrsim 1
$$

This is just a more formal restatement of our discussion in the preceding paragraph: given $\left|\mathfrak{M}^{\text {osc }}\right|_{\max } \sim 1$, the instability condition is satisfied all the way to very large internal fields corresponding to $\nu \sim 1$. This is unlike the ordinary metals where the largest magnetic field that allows such an instability is typically much smaller. As explained in Appendix D, the origin of this is the very small internal gauge field stiffness, which is implicitly built into the numerical estimate $\left|\mathfrak{M}^{\text {osc }}\right|_{\max } \sim 1$ in the above formalism. However, such large numerical value of $\left|\mathfrak{M}^{\text {osc }}\right|_{\max }$ is unavoidable since it is the same mean-field energetics that stabilizes the Fermi sea state and determines both the gauge field stiffness and the spinon kinetic energy.

\section{Mean-field description at finite temperature}

The preceding analysis was performed at zero temperature. Also, we ignored the Zeeman spin coupling to the magnetic field. We first note that for integer filling $\nu$ the spinon spectrum has a gap given roughly by the corresponding cyclotron frequency. Such an integer quantum Hall state of spinons is in fact a chiral spin liquid ${ }^{18}$ induced by the magnetic field, and the above treatment suggests a sequence of first-order transitions stepping through such states rather than a continuous variation of $\phi^{\text {int }}$ (see Fig. 3). Finite temperature smears the effects of the discreteness of the Landau levels, and the instability of the flux states with continuously varying $\phi^{\text {int }}$ becomes weaker.

To proceed more quantitatively, we again use mean field and apply standard magneto-oscillation results at finite temperature in two dimensions supplemented with our estimates of the effective parameters such as the cyclotron frequency Eq. (17). Thus, we can roughly incorporate the effects of the finite temperature and the Zeeman spin splitting by multiplying the oscillating magnetization $\mathfrak{M}^{\text {osc }}$ by the corresponding suppression factors $R_{T}$ and $R_{S}$ from Eq. (D2) in Appendix D. The instability condition Eq. (23) then reads

$$
R_{T} R_{S} \nu^{2}\left|\mathfrak{M}^{\text {osc }}\right|_{\max } \gtrsim 1 .
$$

For a fixed external magnetic field, we can use Eq. (13) to estimate the corresponding spinon filling $\nu$ in the internal gauge field. Focusing on the temperature suppression factor, the homogeneous flux state becomes unstable when the temperature is below

$$
T_{\text {instab }}=\frac{x \hbar \omega_{c}}{2 \pi^{2}}, \quad \frac{\sinh (x)}{x} \approx \nu^{2}\left|\mathfrak{M}^{\text {osc }}\right|_{\max } .
$$

For high filling factors $\nu$ and using $\left|\mathfrak{M}^{\text {osc }}\right|_{\max } \sim 1$, we estimate with $\operatorname{logarithmic}$ accuracy $x \sim 2 \log (\nu)$. We give numerical estimates for the $\kappa-(\mathrm{ET})_{2} \mathrm{Cu}_{2}(\mathrm{CN})_{3}$ material in Sec. IV.

Let us briefly think what happens beyond the mean field. The chiral spin liquid states are stable topologically ordered phases $^{18}$ at zero temperature, but strictly speaking do not survive on long length scales at any nonzero temperature in two dimensions. Instead, the spin system can be continuously connected to the featureless high-temperature paramagnet. In this case, we interpret the mean-field estimate Eq. (25) as delineating the temperature-field regime in which the spin system becomes particularly "soft" in its response to the magnetic field. It is also possible for a $T=0$ first-order transition between chiral states to extend to small finite temperatures even though the paramagnetic phases on both sides do not have a symmetry or topological distinction any more. Note also that in the above schematic treatment, we have not considered the temperature dependence of the effective parameters [for example, even in the formal mean field, the effective $t^{\text {spinon }}$ decreases with temperature and disappears above $T \sim 50-100 \mathrm{~K}$ for the $\kappa-(\mathrm{ET})_{2} \mathrm{Cu}_{2}(\mathrm{CN})_{3}$ parameters]. Such detailed questions on the behavior of the spin system at finite temperature remain open for future investigations.

\section{E. Inhomogeneous state in the physical system at low temperatures}

We conclude the mean-field description with a discussion of some experimentally relevant aspects. Our analysis indicates the instability of the homogeneous flux states with continuously varying $\phi^{\text {int }}$ at temperatures below $T_{\text {instab. Through- }}$ out, we treat the external magnetic field as fixed, and the instability represents a strong nonlinear back action of the spinons onto the internal gauge field in the presence of such fixed external source. Treating the magnetic field as fixed is justified since, as we describe in Appendixes C and D, the electromagnetic field is six to seven orders of magnitude more stiff than the internal gauge field and will not adjust itself to the electronic system until much lower temperatures. Of course, there is a slight effect on the local magnetic field depending on the state of the spin system, but for example for the uniform flux mean field state we estimate the orbital contribution to be one order of magnitude smaller than the Pauli spin contribution. The physical setting therefore has fixed $\Phi^{\text {ext }}$. In particular, we do not have access to different sample "demagnetization geometries" considered for the magnetic interaction phenomenon in conventional metals ${ }^{11,12}$ - the internal gauge field does not "leak out" of the sample. 
In such setting in an ideal crystal system at zero temperature, we predict a sequence of first-order transitions, ${ }^{19}$ but each phase is still a uniform flux state with the corresponding integer $\nu$. This situation, however, is highly susceptible to large-scale imperfections of a real system, and an outcome with many domains is likely. Another important consideration is the possible crystal mosaic in the sample, since the discussed orbital effects are determined by the component of the magnetic field $H_{\perp}$ normal to the two-dimensional plane. A detailed characterization of possible inhomogeneities clearly requires much more material knowledge.

\section{APPLICATION TO $\kappa-(\mathrm{ET})_{2} \mathrm{Cu}_{2}(\mathrm{CN})_{3}$}

Throughout, we have used the $\kappa-(\mathrm{ET})_{2} \mathrm{Cu}_{2}(\mathrm{CN})_{3}$ parameters to motivate various approximations and make the otherwise formal discussion more physical. Here we want to focus more on the material itself and collect the relevant numbers in one place.

We first want to point out that the material is rather clean. Thus, we estimate $k_{F} l \geq 50$ in the metallic phase at $0.8 \mathrm{GPa}$. Shubnikov-de Haas $(\mathrm{SdH})$ oscillations are observed ${ }^{20}$ at temperatures around $1.5 \mathrm{~K}$, which is another indication of the material quality. The $\mathrm{SdH}$ signal is consistent with the Fermi surface and the one-band model description. For reference, the Landau level filling factor for the $\kappa$ - $(\mathrm{ET})_{2} \mathrm{Cu}_{2}(\mathrm{CN})_{3}$ material is given by $\nu^{\mathrm{ext}}=3595 / H(\mathrm{~T})$. In Appendix D, we estimate the characteristic magnetic field $H_{0} \approx 5 \mathrm{~T}$ and temperature $T_{\mathrm{dm}} \approx 0.16 \mathrm{~K}$ for the magnetic interaction instability ${ }^{21-23}$ in this metallic phase coupled to the electromagnetic field.

Turning to the spin liquid phase, in Ref. 9 we used the low-temperature susceptibility to estimate the spinon hopping amplitude $t_{\text {spinon }} \simeq 350 \mathrm{~K}$. We can also use the meanfield estimate Eq. (23), which gives a smaller but reasonable value $t_{\text {spinon }} \simeq 100 \mathrm{~K}$. Using Eqs. (17) and (25), and $\nu^{\text {int }}$ $=\nu^{\mathrm{ext}} / \gamma$ with $\gamma \simeq 1-2$, we obtain the characteristic temperature at $B=8 \mathrm{~T}$ to be $T_{\mathrm{instab}} \simeq 1-2 \mathrm{~K}$. Above this temperature, we expect uniform flux state.

We now summarize qualitative predictions for the inhomogeneous state induced by large magnetic fields at low temperatures below $T_{\text {instab }}$. As discussed in the paragraph following Eq. (22), the characteristic field $H_{0}$ for the spinon system is beyond any practical fields, and we predict that in the laboratory fields the instability temperature will increase roughly linearly with the applied field, Eq. (25). Manifestations of this instability become more pronounced with increasing field and decreasing temperature below $T_{\text {instab. Since }}$ this is an orbital effect, we expect distinction for fields perpendicular and parallel to the conducting planes. One consequence of this scenario is that magneto-oscillation measurements in their usual sense cannot be used to detect the spinon Fermi surface in the spin liquid state considered here. This is because the regime where such oscillations can become visible is likely preempted by the instability of the homogeneous state. Since the instability is in some sense an extreme manifestation of the magneto-oscillations, conditions of the magnetic field uniformity and the crystal mosaic are important experimental considerations, as discussed in Sec. III E.
Other measurements in the spin liquid phase performed in high magnetic field and at low temperature may also require more careful interpretation.

\section{CONCLUSIONS}

The main content of this work was summarized in the introduction, and possible application to the $\kappa$-(ET) ${ }_{2} \mathrm{Cu}_{2}(\mathrm{CN})_{3}$ experiments including some numerical estimates was discussed in the preceding section. Here we want to conclude with a more general point. While there has been a significant progress in the theoretical understanding of exotic quantum phases, the lack of the material evidence is viewed as a major obstacle. Recently, several candidate spin liquid systems have been found in frustrated spin systems, ${ }^{1,24,25}$ of which the $\kappa-(\mathrm{ET})_{2} \mathrm{Cu}_{2}(\mathrm{CN})_{3}$ is a very promising example. This material motivated and guided the detailed theoretical considerations in the present work on the magnetic field response of the spin liquid state stabilized near the Mott transition. Without such a guide, the proposed effects would be hard to anticipate. More work matching experiment and theory focusing on material properties will likely be fruitful in developing our understanding and in further pursuits of such unusual quantum phases. One specific motivation for the present study has been the anomalous NMR line broadening reported in Refs. 3 and 5. While some of our expectations for the inhomogeneous state at low temperatures resemble the experimental phenomenology, the gradual development of the broadening starting from rather high temperatures and also the magnitude of the inhomogeneous fields are more suggestive of a stronger impurity mechanism. Experiments in parallel field can further clarify the role of the impurity and orbital effects. The main predictions in the present paper of the spinon orbital field and the fragility of the spinon Fermi sea state in the applied magnetic field represent intrinsic response of the spin liquid and are expected to play an important role at low temperatures. It is hoped that our proposals will stimulate further experimental and theoretical questions.

Note added. Recently, the author learned of Ref. 28, which also discusses the coupling of the external field to the spin chirality and considers possible effects in some different contexts for Hubbard systems with triangles.

\section{ACKNOWLEDGMENTS}

The author has benefited from useful discussions with $\mathrm{M}$. P. A. Fisher, V. Galitski, Y.-B. Kim, P. A. Lee, S. S. Lee, A. Paramekanti, T. Senthil, and A. Vishwanath, and also thanks the Aspen Center for Physics Summer Program on "Gauge Theories in Condensed Matter Physics" where part of this work was completed. The research at KITP is supported through NSF Grant No. PHY-9907949. Use of HewlettPackard and CNSI Computer Facilities at UCSB is acknowledged.

\section{APPENDIX A: HUBBARD TO HEISENBERG HAMILTONIAN IN THE PRESENCE OF MAGNETIC FIELD}

This appendix gives the effective spin Hamiltonian to or$\operatorname{der} t^{4} / U^{3}$ for a general lattice Hubbard system with complex 


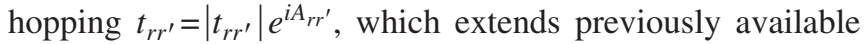
results $^{26}$ to the case in the presence of the magnetic field.

The starting point is the Hubbard Hamiltonian at half filling

$$
H=U \sum_{r} n_{r \uparrow} n_{r \downarrow}-\sum_{r r^{\prime}} t_{r r^{\prime}} c_{r \sigma}^{\dagger} c_{r^{\prime} \sigma},
$$

with $t_{r^{\prime} r}=t_{r r^{\prime}}^{*}$. The hopping part is treated as a perturbation, and a canonical transformation is performed into the sector with no double occupancy. The effective Hamiltonian to or$\operatorname{der} t^{4} / U^{3}$ reads

$$
\begin{gathered}
H=U \sum_{r} n_{r \uparrow} n_{r \downarrow}-\sum_{r r^{\prime}} t_{r r^{\prime}} c_{r \sigma}^{\dagger} c_{r^{\prime} \sigma}, \\
H_{2}=\sum_{\bullet \bullet} \frac{2\left|t_{12}\right|^{2}}{U}\left(P_{12}-1\right), \\
H_{3}=\sum_{\bullet} \frac{6 \operatorname{Im}\left(t_{12} t_{23} t_{31}\right)}{U^{2}} i\left(P_{123}-\text { H.c. }\right), \\
H_{4}=\sum_{\bullet \rightarrow} \frac{8\left|t_{12}\right|^{4}}{U^{3}}\left(1-P_{12}\right)+\sum_{1,2,3}^{\prime} \frac{\left|t_{12}\right|^{2}\left|t_{13}\right|^{2}}{U^{3}}\left(P_{23}-1\right) \\
+\sum_{\bullet=} \frac{\operatorname{Re}\left(t_{12} t_{23} t_{34} t_{41}\right)}{U^{3}}\left[20\left(P_{1234}+\text { H.c. }\right)\right. \\
\left.-12\left(P_{12}+P_{23}+P_{34}+P_{41}+P_{13}+P_{24}\right)+32\right] .
\end{gathered}
$$

Here $P_{12}, P_{123}$, and $P_{1234}$ denote two-spin, three-spin, and four-spin exchange operators respectively. The latter two operators move the spins in a ringlike manner. For example, the three-spin exchange in terms of the fermions is $P_{123}$ $=\left(c_{1 \alpha}^{\dagger} c_{1 \beta}\right)\left(c_{2 \beta}^{\dagger} c_{2 \gamma}\right)\left(c_{3 \gamma}^{\dagger} c_{3 \alpha}\right)$, and acts on the spins as $P_{123}:\left|\sigma_{1}, \sigma_{2}, \sigma_{3}\right\rangle \rightarrow\left|\sigma_{3}, \sigma_{1}, \sigma_{2}\right\rangle$. To simplify the final expressions, we used the identities Eqs. (4) and (5) specific to the spin- $1 / 2$ case.

The sum in $\mathrm{H}_{2}$ is over all bonds of the lattice. The sum in $\mathrm{H}_{3}$ is over all three-site loops, where each group of three sites connected by the links enters the sum precisely once. Similarly, the sum in the second line for $H_{4}$ is over four-site loops, which are counted with no base point or orientation. Finally, the primed sum in the first line for $H_{4}$ is over distinct sites $1,2,3$ with nonzero $t_{12}$ and $t_{13}$ (each contribution will therefore appear two times). Equation (1) in the main text is obtained by specializing to the isotropic triangular lattice.

Note that the complex phase of a loop product of $t_{r r^{\prime}}$ measures the flux of the magnetic field through the loop. Without such fluxes, $H_{3}$ vanishes and after some transformations we reproduce the result of Ref. 26.

As a side remark, we observe that in the absence of threesite loops, there is no linear coupling to the magnetic field to this order in $t / U$. One can also show that for a half-filled Hubbard model on a bipartite lattice, the effective Hamiltonian in the presence of the magnetic field can only contain terms that are even in the applied field.

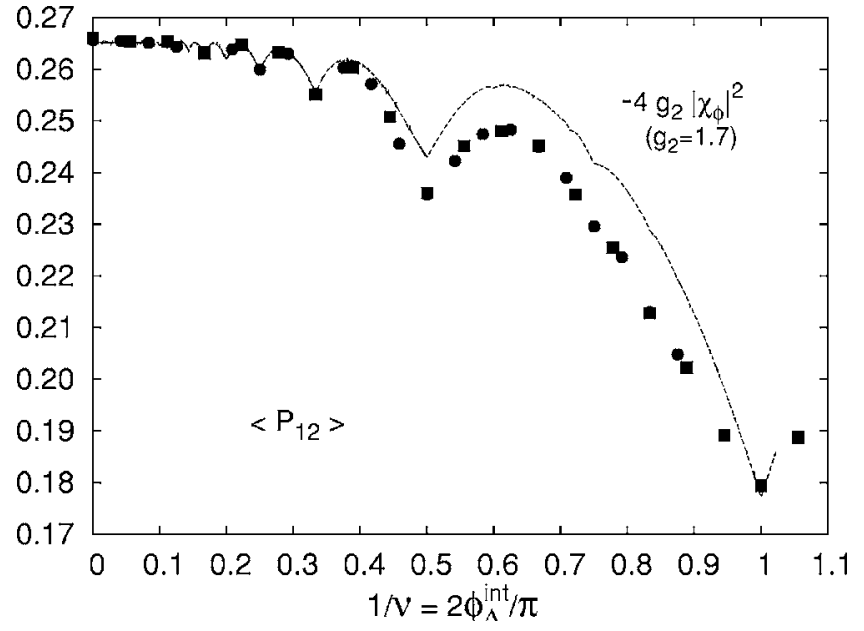

FIG. 4. Expectation value of the two-spin exchange $P_{12}$ on each link evaluated in the Gutzwiller-projected uniform flux state. The data are for commensurate fluxes on triangular lattice cuts with sizes $36 \times 12$ (squares) and $48 \times 12$ (circles) with periodic boundary conditions. The line shows the renormalized mean-field estimate with $g_{2}=1.7$ (the mean-field data are obtained using Fig. 2). Since we are interested only in the variation with $\phi^{\text {int }}$, a constant offset is added to the mean-field values.

\section{APPENDIX B: RENORMALIZED MEAN FIELD}

In this appendix, we estimate the renormalization factors $g_{2}, g_{4}$, and $g_{3}$ used in the mean-field treatment in Sec. III. We consider the uniform flux states and measure the relevant expectation values of $P_{12},\left(P_{1234}+\right.$ H.c. $)$, and $i\left(P_{123}-\right.$ H.c. $)$ in the Gutzwiller-projected wave functions. The variation of the expectation values with $\phi^{\text {int }}$ is matched with the corresponding mean-field estimates-see Eq. (9). Figures 4-6 show this matching and are self-explanatory. We note that the Gutzwiller wave function evaluations can be performed only for small system sizes and large values of $\phi^{\text {int }}$. However, we expect the same trend to remain for small fluxes as well. For small fluxes, an analytical mean-field treatment is then performed (Sec. III) using the estimated renormalization factors.

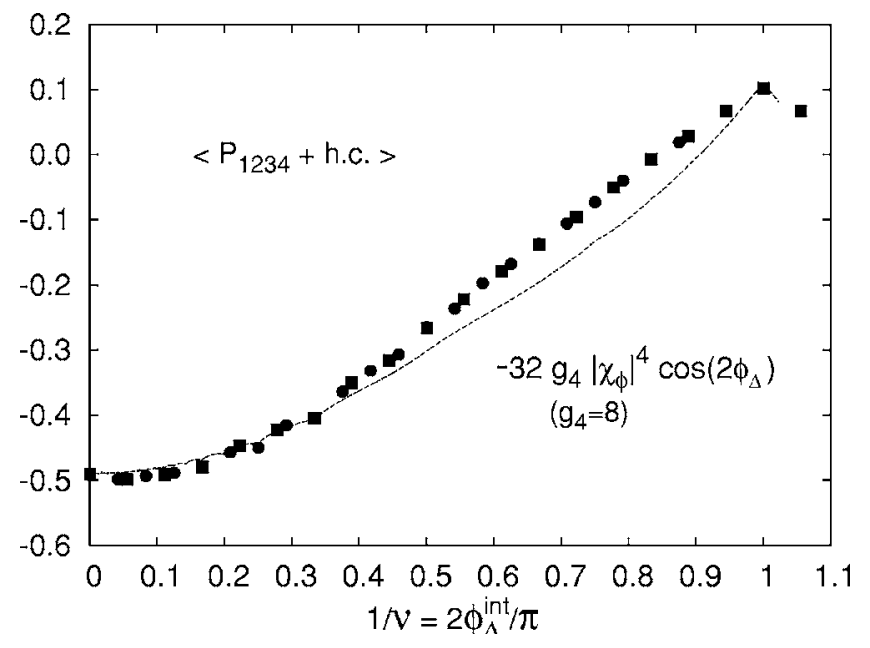

FIG. 5. Same as in Fig. 4, but for the ring exchange operator $P_{1234}+$ H.c. around a rhombus. 


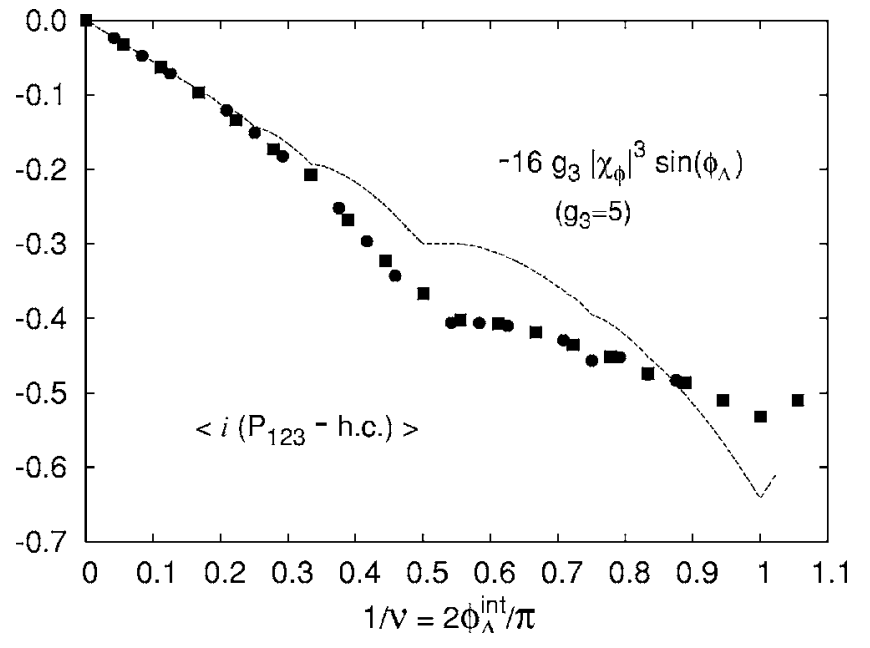

FIG. 6. Same as in Fig. 4, but for the three-spin operator $i\left(P_{123}-\right.$ H.c. $)$ around an elementary triangle. The orientation of the triangular loop $1 \rightarrow 2 \rightarrow 3 \rightarrow 1$ coincides with that of $\phi_{123}^{\text {int }}=\phi_{\Delta}^{\text {int }}$.

This is the main idea of the so-called renormalized meanfield approach. ${ }^{27}$

\section{APPENDIX C: SPINON-GAUGE THEORY}

In the main text, we often use the gauge theory language, ${ }^{14}$ and it is useful to state the connection more explicitly. We follow the treatment of Ref. 10 as an example. One starts with a slave-rotor representation $c_{r \sigma}^{\dagger}=f_{r \sigma}^{\dagger} e^{i \theta_{r}}$ of the Hubbard model and obtains the following effective theory for the spinons $f_{r \sigma}$ and chargons $e^{i \theta_{r}}$ :

$$
\begin{gathered}
S=\int d \tau\left(\mathcal{L}_{f}+\mathcal{L}_{\theta}\right), \\
\mathcal{L}_{f}=\sum_{r} f_{r \sigma}^{\dagger}\left(\partial_{\tau}-i a_{r}^{0}-\mu\right) f_{r \sigma}-\sum_{r r^{\prime}} t_{f} e^{i a_{r r^{\prime}} f_{r \sigma}^{\dagger} f_{r^{\prime} \sigma},} \\
\mathcal{L}_{\theta}=\frac{1}{2 U} \sum_{r}\left(\partial_{\tau} \theta_{r}-a_{r}^{0}\right)^{2}-\sum_{r r^{\prime}} t_{\theta} e^{i\left(\theta_{r}-\theta_{r^{\prime}}-a_{r r^{\prime}+A_{r r^{\prime}}}^{\mathrm{ext}}\right)}
\end{gathered}
$$

The imaginary time is continuous, while the space lattice is retained to indicate similarity of the spinon part with that in Eq. (8). The spinons and the chargons are coupled with opposite charge to the internal gauge field $\left(\boldsymbol{a}, a^{0}\right)$, whose spatial components represent fluctuations of the phase of the spinon hopping field, $t_{r r^{\prime}}^{\text {spinon }}=t_{f} e^{i a_{r r^{\prime}}}$, while the temporal $a_{r}^{0}$ implement the slave-particle constraints. The coupling to the external field $A^{\text {ext }}$ is included schematically by assigning the electron charge to the chargon field.

Assuming the chargons are gapped and integrating them out, we are left with the spinon-gauge system where the gauge field now has some stiffness $\kappa$,

$$
\mathcal{L}_{a}=\frac{\kappa}{2}\left(\boldsymbol{\nabla} \wedge \mathbf{a}-\boldsymbol{\nabla} \wedge \mathbf{A}^{\mathrm{ext}}\right)^{2}+\frac{\kappa_{\tau}}{2}\left(\partial_{\tau} \mathbf{a}-\boldsymbol{\nabla} a^{0}\right)^{2} .
$$

Here $\boldsymbol{\nabla} \wedge \boldsymbol{a}=\partial_{x} a_{y}-\partial_{y} a_{x} \equiv b$; we separated this "spatial" part for later convenience.
Our mean-field treatment Eq. (9) roughly corresponds to considering the spinon-gauge system with static but possibly spatially varying gauge field $\boldsymbol{a}$. Observe that the internal gauge field obtains its stiffness after integrating out the massive chargons, and also observe how the magnetic field couples to the internal gauge field. This is similar to our discussion of the effective spin Hamiltonian, where the crucial ingredient is also charge fluctuations. Note however that the effective Hamiltonian treatment is more explicit. Thus, the gauge field stiffness originates primarily from the fourspin ring exchanges, while the coupling to the magnetic field comes from the three-spin processes-see Eqs. (9) and (18). In the schematic gauge theory derivation this distinction is not being made. The result $\gamma=1$ of such schematic treatment roughly agrees with our estimate using Eq. (13), but this is rather fortuitous - e.g., as we mention toward the end of Appendix $\mathrm{A}$, in a Hubbard model on a lattice with no elementary triangles the linear coupling of the internal gauge flux to the applied magnetic field does not appear even to order $t^{4} / U^{3}$. To capture the detail present in our Eq. (13) in the gauge theory approach, one needs to go back to the original slave particle rewriting and carefully consider saddle point conditions and integrations over massive fields when deriving Eq. (C1) in the presence of the external field. As an example, the suppression of the gauge field stiffness due to the proximity to the flux phase discussed after Eq. (13) originates from some modes which become soft near the flux phase. The effective spin Hamiltonian approach is more transparent in this respect and also allows quantitative estimates of the physical quantities.

In particular, from Eq. (20) we quote the internal gauge field stiffness in terms of the energy cost per triangular lattice site, $\epsilon_{\text {int }}=\kappa_{4}\left(\phi_{\Delta}^{\text {int }}\right)^{2}$,

$$
\kappa_{4} \sim 192 g_{4} J_{4}\left|\chi_{0}\right|^{4} \sim 0.14 g_{4} J_{4} \sim 2 \mathrm{meV} .
$$

Here we used the Sec. II estimate $J_{4} \simeq 2 \mathrm{meV}$, and used the Fig. 5 estimate $g_{4} \simeq 8$.

This can be compared with the corresponding stiffness of the physical electromagnetic (EM) field in the bulk of the $\kappa-(\mathrm{ET})_{2} \mathrm{Cu}_{2}(\mathrm{CN})_{3}$ compound, $\epsilon_{\mathrm{EM}}=V B^{2} /(8 \pi) \equiv \kappa_{\mathrm{EM}}\left(\Phi_{\Delta}^{\mathrm{ext}}\right)^{2}$,

$$
\kappa_{\mathrm{EM}}=\frac{V \hbar^{2} c^{2}}{8 \pi A_{\Delta}^{2} e^{2}}=1.1 \times 10^{4} \mathrm{eV} .
$$

Here $V=850.6 \AA^{3}$ is the three-dimensional (3D) volume per triangular lattice site, $A_{\Delta}=28.76 \AA^{2}$ is the area of an elementary triangle, and $\Phi_{\Delta}^{\mathrm{ext}}=e B A_{\Delta} /(\hbar c)$ is the appropriate dimensionless external flux.

We therefore conclude that the bare internal gauge field stiffness is $\sim 10^{7}$ times smaller than the electromagnetic field stiffness. As we discuss in the following Appendix D, this makes a dramatic difference in the response of the spinongauge system to the external field compared with that of electrons in a conventional metal.

\section{APPENDIX D: ANALOGY WITH MAGNETIC INTERACTION EFFECTS IN METALS}

The purpose of this appendix is to clarify the status of our mean-field treatment in Sec. III by pointing out the analogy 
with the so-called magnetic interaction effects in the study of magneto-oscillations in metals. This material is available in textbooks. ${ }^{11,12}$ The presentation below parallels some of our discussion in Sec. III in a different language, but we hope that the reader will benefit from this duplication.

For ease of reference, we first write down the oscillating part of the magnetization of a two-dimensional electron gas in a field $H$ at a finite temperature $T$ :

$$
\mathcal{M}^{\mathrm{osc}}(H)=R_{T} R_{S} \frac{n e \hbar}{\pi m^{*} c} \sin \left(\frac{2 \pi F}{H}\right) .
$$

Only the main harmonic is shown. The magnetization is given per unit area, and $n$ is the $2 \mathrm{D}$ electron density (including spin); $m^{*}$ is the cyclotron mass; $F=n \pi c \hbar / e$ so that $F / H=\nu$ is the Landau level filling for each spin species. The suppression factors $R_{T}$ and $R_{S}$ due to finite temperature and Zeeman spin splitting are

$$
R_{T}=\frac{2 \pi^{2} T / \hbar \omega_{c}}{\sinh \left(2 \pi^{2} T / \hbar \omega_{c}\right)}, \quad R_{S}=\cos \frac{g \pi m^{*}}{2 m_{e}},
$$

where $g$ is the spin $g$ factor.

The essence of the magnetic interaction effects is the back action of the electrons onto the electromagnetic field, which can be significantly enhanced by the oscillating character of $\mathcal{M}$. Specifically, consider fermions coupled to a dynamical gauge field $a$ in the presence of the external field $A^{\text {ext }}$, with the Lagrangian density $\mathcal{L}_{f}+\mathcal{L}_{a}$ given by Eqs. (C1) and (C2). The microscopic magnetic field is $b=\nabla \times a$ and the external field is $H^{\mathrm{ext}}=\nabla \times A^{\mathrm{ext}}$. In the case of electrons, $a$ represents the physical electromagnetic field and $\kappa$ is the appropriate EM stiffness; e.g., in a 2D layered material the magnetic field energy per unit area is $d^{*} b^{2} / 8 \pi$ where $d^{*}$ is the spacing between the layers.

The key observation is that electrons see the average microscopic field $B$ because the typical Larmor orbits are large. The mean-field treatment is then to assume a static but possibly spatially varying such field $B(r)$ and solve the Landau problem for electrons moving in this field. The mean-field functional including the energy of the gauge field is ${ }^{11-13}$

$$
\Omega_{\mathrm{mf}}=\Omega_{\mathrm{ferm}}(B)+\frac{\kappa}{2}\left(B-H^{\mathrm{ext}}\right)^{2},
$$

where $\Omega_{\text {ferm }}(B)$ is the free energy density for fermions in the static field $B(r)$. The mean-field functional is to be minimized with respect to $B(r)$. If we look for a uniform solution, we obtain

$$
H^{\mathrm{ext}}=B+\frac{1}{\kappa} \frac{\partial \Omega_{\mathrm{ferm}}}{\partial B} \equiv B-\frac{1}{\kappa} \mathcal{M}(B),
$$

where in the last equation we defined the magnetization density $\mathcal{M}(B)$. For a $2 \mathrm{D}$ electron gas, the oscillating piece of the magnetization is given in Eq. (D1), and the mean-field treatment is to replace $H \rightarrow B$.

Equation (D4) is to be solved for $B$. It is customary to plot the right-hand side as a function of $B$ and seek the intersection with the horizontal line $H^{\text {ext }}$. When this is unique, the corresponding uniform field is the sought for stable solution. However, $\mathcal{M}(B)$ contains an oscillating piece, and if we find that

$$
\frac{1}{\kappa} \frac{\partial \mathcal{M}}{\partial B}>1
$$

then the solution is no longer unique, which signals an instability. Note that while the amplitude of the oscillations $|\mathcal{M}|_{\max }(T=0)=n e \hbar /\left(2 m^{*} c\right)$ is small in metals compared to typical $\kappa H$, the amplitude of the "susceptibility" $\partial \mathcal{M} / \partial B$ obtains an additional factor $\sim F / H^{2}$, and the instability condition can be satisfied at low temperatures and not too large fields. After simple transformations, the instability condition reads

$$
R_{T} R_{S} n \frac{\hbar^{2} k_{F}^{2}}{2 m^{*}} \geq \frac{\kappa}{2} H^{2}
$$

where $k_{F}$ is the Fermi wave vector. When this condition is satisfied, the gain in the fermion energy when the field $B$ is adjusted to obtain integer Landau level filling overweighs the magnetic field energy cost. Since $R_{T}$ and $R_{S}$ do not exceed 1, the above equation sets the maximal value $H_{0}$ for the instability to occur at zero temperature. On the other hand, at a finite temperature, we also require the Landau levels to be resolved, which is determined by $R_{T}$. Roughly, for fields of order $H_{0}$, we require $2 \pi^{2} T \lesssim \hbar \omega_{c}$ with $\omega_{c}$ set by $H_{0}$.

The 2D Landau problem for free electrons can be solved beyond the above single-harmonic treatment, and Ref. 21 contains such expressions for the phase boundary of the instability region. For example, the field $H_{0}$ is determined from

$$
n \frac{\hbar^{2} k_{F}^{2}}{2 m^{*}}=\kappa H_{0}^{2}=d^{*} \frac{H_{0}^{2}}{8 \pi} .
$$

Reference 21 also contains formulas directly appropriate for the layered organic materials. As a numerical application, consider the metallic phase of the $\kappa-(\mathrm{ET})_{2} \mathrm{Cu}_{2}(\mathrm{CN})_{3}$ compound obtained under pressure of $0.76 \mathrm{GPa}$, in which magneto-oscillations were reported in Ref. 20. Using $\epsilon_{F}$ $\approx 100 \mathrm{meV}$, the cyclotron mass ratio $\eta_{c}=m^{*} / m_{e} \approx 4$, the interlayer spacing $d^{*}=14.84 \AA^{2}$, and ignoring sample demagnetization effects, we estimate $H_{0} \approx 5 \mathrm{~T}$; the peak temperature on the phase boundary of the instability regime is estimated to be $T_{\mathrm{dm}} \approx 0.16 \mathrm{~K}$.

Various aspects of what happens once the homogeneous state becomes unstable are discussed in textbooks. ${ }^{11,12}$ There is also a growing recent literature on the observation of Condon domains ${ }^{22}$ in metals; see Ref. 23 and references therein.

The point that we want to make is that our mean-field treatment in Sec. III has the same character as the described treatment of the magnetic interaction effects in metals. Thus, our mean-field energy Eq. (18) can be readily identified with Eq. (D3), while the self-consistency condition Eq. (22) corresponds to Eq. (D4). Clearly, the treatment of the magnetic interaction effects requires taking into account the energy of the gauge field. This is implicit in our mean-field treatment of Sec. III. At this mean-field stage, we do not need to dis- 
entangle the gauge field energy from the total energy and worry about the emergent nature of the gauge field, and this makes the procedure more simple.

An important difference between the spinon-gauge system and the electrons in metals coupled to the EM field is the very small gauge field stiffness in the spinon case, as we estimated in Appendix C. Because of this, the instability condition is satisfied more readily in the spinon-gauge system. Thus, because the internal gauge field stiffness is $10^{6}-10^{7}$ times smaller while the kinetic parameters of spinons are not far from those of electrons, the above estimate of $H_{0}$ is to be multiplied by a factor of order $10^{3}$. This is an impractically large field, and the spinon-gauge system in the laboratory is in the regime of much smaller fields. In this regime, the temperature at which the instability occurs in a given field $H$ can be estimated as

$$
T_{\text {instab }}=\frac{x \hbar \omega_{c}}{2 \pi^{2}}, \quad \frac{\sinh (x)}{x} \approx \frac{n \hbar^{2} k_{F}^{2} / m^{*}}{\kappa H^{2}} .
$$

This temperature depends on the stiffness only logarithmically, but as numerical estimates in Sec. III show, even under the logarithm the seven orders of magnitude difference in the relevant stiffnesses produces a much wider temperature range for the instability in the spinon-gauge system.
${ }^{1}$ Y. Shimizu, K. Miyagawa, K. Kanoda, M. Maesato, and G. Saito, Phys. Rev. Lett. 91, 107001 (2003).

${ }^{2}$ Y. Kurosaki, Y. Shimizu, K. Miyagawa, K. Kanoda, and G. Saito, Phys. Rev. Lett. 95, 177001 (2005).

${ }^{3}$ Y. Shimizu, K. Miyagawa, K. Kanoda, M. Maesato, and G. Saito, Phys. Rev. (to be published).

${ }^{4}$ T. Komatsu, N. Matsukawa, T. Inoue, and G. Saito, J. Phys. Soc. Jpn. 65, 1340 (1996).

${ }^{5}$ A. Kawamoto, Y. Honma, and K. I. Kumagai, Phys. Rev. B 70, 060510(R) (2004).

${ }^{6}$ R. H. McKenzie, Comments Condens. Matter Phys. 18, 309 (1998).

${ }^{7}$ M. Imada, T. Mizusaki, and S. Watanabe, cond-mat/0307022 (unpublished); H. Morita, S. Watanabe, and M. Imada, J. Phys. Soc. Jpn. 71, 2109 (2002); J. Liu, J. Schmalian, and Nandini Trivedi, Phys. Rev. Lett. 94, 127003 (2005).

${ }^{8}$ W. LiMing, G. Misguich, P. Sindzingre, and C. Lhuillier, Phys. Rev. B 62, 6372 (2000).

${ }^{9}$ O. I. Motrunich, Phys. Rev. B 72, 045105 (2005).

${ }^{10}$ S.-S. Lee and P. A. Lee, Phys. Rev. Lett. 95, 036403 (2005).

${ }^{11}$ D. Shoenberg, Magnetic Oscillations in Metals (Cambridge University Press, Cambridge, U.K., 1984).

${ }^{12}$ A. A. Abrikosov, Fundamentals of the Theory of Metals (NorthHolland, Amsterdam, 1988).

${ }^{13}$ T. Holstein, R. E. Norton, and P. Pincus, Phys. Rev. B 8, 2649 (1973).

${ }^{14}$ For a recent review of gauge theories of strongly correlated matter, see P. A. Lee, N. Nagaosa, and X.-G. Wen, Rev. Mod. Phys. 78, 17 (2006), and references therein.

${ }^{15}$ X.-G. Wen, Phys. Rev. B 65, 165113 (2002); Y. Zhou and X.-G. Wen, cond-mat/0210662 (unpublished).

${ }^{16}$ Other chiral spin liquid states such as a Gutzwiller-projected $d$ $+i d$ state can also improve the trial energy in the presence of the magnetic field. The present work focuses on the flux states for their simplicity, but the detailed energetics including other competing states has not been explored.
${ }^{17}$ I. Affleck and J. B. Marston, Phys. Rev. B 37, R3774 (1988); Y. Hasegawa, P. Lederer, T. M. Rice, and P. B. Wiegmann, Phys. Rev. Lett. 63, 907 (1989); E. H. Lieb, ibid. 73, 2158 (1994).

${ }^{18}$ X. G. Wen, F. Wilczek, and A. Zee, Phys. Rev. B 39, 11413 (1989).

${ }^{19} \mathrm{An}$ interesting possibility discussed in the literature (see. e.g., Ref. 12 and references therein) is a "true" inhomogeneous flux state that would be inhomogeneous also in an ideal crystal and in any geometry. The analysis predicting a sequence of firstorder transitions assumes that at such transition the energy of a domain wall between the two neighboring phases is positive. If, on the other hand, the domain wall energy turns out to be negative, then the different phases want to mix and instead the true ground state has an inhomogeneous flux distribution, possibly modulated at some wave vector. This interesting scenario, which is usually believed to be less likely, has not been explored in the present work.

${ }^{20}$ E. Ohmichi, H. Ito, T. Ishiguro, T. Komatsu, and G. Saito, J. Phys. Soc. Jpn. 66, 310 (1997); E. Ohmichi, H. Ito, T. Ishiguro, G. Saito, and T. Komatsu, Phys. Rev. B 57, 7481 (1998).

${ }^{21}$ M. A. Itskovsky, T. Maniv, G. F. Kventsel, and I. D. Vagner, Phys. Rev. B 55, 5636 (1997).

22 J. H. Condon, Phys. Rev. 145, 526 (1966).

${ }^{23}$ R. B. G. Kramer, V. S. Egorov, V. A. Gasparov, A. G. M. Jansen, and W. Joss, Phys. Rev. Lett. 95, 267209 (2005); A. Gordon, I. D. Vagner, and P. Wyder, Adv. Phys. 52, 385 (2003). V. S. Egorov, cond-mat/0505415 (unpublished).

${ }^{24}$ R. Coldea, D. A. Tennant, and Z. Tylczynski, Phys. Rev. B 68, 134424 (2003).

${ }^{25}$ A. Fukaya et al., Phys. Rev. Lett. 91, 207603 (2003); Z. Hiroi et al., J. Phys. Soc. Jpn. 70, 3377 (2001).

${ }^{26}$ M. Takahashi, J. Phys. C 10, 1289 (1977); A. H. MacDonald, S. M. Girvin, and D. Yoshioka, Phys. Rev. B 37, 9753 (1988).

${ }^{27}$ F. C. Zhang, C. Gros, T. M. Rice, and H. Shiba, Semicond. Sci. Technol. 1, 36 (1988); C. Gros, Ann. Phys. (N.Y.) 189, 53 (1989).

${ }^{28}$ Diptiman Sen and R. Chitra, Phys. Rev. B 51, 1922 (1995). 